\title{
Shift Force Loading Rules Research for Automated Mechanical Transmission
}

\author{
Li Bo, Ge Wenqing*, Zhao Yiqiang and Chen Shanshi
}

School of Transporation and Vehicle Engineering, Shandong University of Technology, Zibo 255049, China

\begin{abstract}
To improve the system reliability and reduce the shift shock of Automated Mechanical Transmission, shift force loading rules is researched on the basis of strength and stiffness analyzing of shift fork. The shift mechanism of A-5speed manual transmission is used as an example to illustrate the simulation, co-relation and validation of the shift fork strength and stiffness. The three-dimensional model of the shift fork is built and the finite element analysis model based on ABAQUS is established. And then, the influence of strength and stiffness of shift fork on the shift force loading rules can be analyzed. The fork tends to deflect with the synchronizer sleeve during synchronization thus acting as a damper and storing energy, the maximum instantaneous force output by driving device under the strength requirement of shift fork is determined. The shift control strategy is corrected according to the stiffness analysis of shift fork. The results shown that, in order to meet the strength requirement of shift fork, the maximum instantaneous force for the 4 gear fork is $1185.5 \mathrm{~N}$. In the same time, the maximum deformation on fork legs is $0.617 \mathrm{~mm}$. The research provides a sufficient theoretical basis for formulating the shift control strategy.
\end{abstract}

Keywords: Maximum instantaneous force, shift force loading rules, shift fork, stiffness, strength.

\section{INTRODUCTION}

The transmission system is one of the crucial systems that affect the vehicle fuel economy [1]. Nowadays, vehicle manufactures put more attention on reducing vehicle fuel consumption due to high fuel price and concerns on global warming. Currently, two main types of transmission used in passenger cars are Manual Transmission (MT) and Automatic Transmission (AT). MT has a high mechanical efficiency and the driver can select gears autonomously. AT is convenient to operate but has relatively higher fuel consumption. Therefore, a type of transmission named Automated Mechanical Transmission (AMT), which combines the advantages of AT and MT, represents a promising solution and is spreading in the recent years [2].

AMT is directly derived from the MT with the integration of servo-actuators into existing devices. The clutch and shift mechanism are controlled automatically by the transmission control unit [3]. But, a poorly designed and controlled AMT may have drawbacks such as prolonged power interruption time and larger shift shock. The poor shifting quality of AMT mainly depends on clutch actuation, but the motion accuracy of shift mechanism cased by the deforming of shift fork will also affect the shifting quality [4]. It is necessary to research the influence of strength and stiffness of shift fork in the process of gear shift.

In reference [5], the stiffness optimization of the shift fork, shift pad height difference calculation and the contact analysis of the shift fork with the synchronizer sleeve have been completed. They establish the result using UG NX Naxtran and then the results have been validated. The components have been modeled using 2nd order tetra elements for better co-relation of the stress and stiffness in the gear shift fork. It has laid a good practical value for optimizing the structure of shift fork. However, the strength and stiffness of shift fork are also important factors that affect the motion accuracy of shift mechanism, and then, AMT will obtain a poor shifting quality.

In order to improve the system reliability and reduce the shift shock of Automated Mechanical Transmission, paper researches the shift force loading rules on the basis of strength and stiffness analyzing of shift fork. Establish the three-dimensional model based on CATIA and the finite element analysis model based on ABAQUS for the shift fork, determine the formulation principle of the maximum instantaneous force, and obtain the maximum instantaneous force output by driving device under the strength requirement of shift fork. The received stiffness analysis result can be used to formulate the shift control strategy. It has a better practical value and prospect for improving the shifting quality.

\section{FORMULATION PRINCIPLE OF MAXIMUM INSTANTANEOUS FORCE}

The requirements of downshift force and gear select torque during the gear shift process are all so small that the influence to shifting quality is nowhere near upshift force. Thus, the shift force loading rules of upshift process is designed focused considering the shifting quality, synchronizer life and drive capability of drive device. The maximum instantaneous force is the primary consideration content for shift control strategy formulating in the process 
of shift force loading rules. While the key impact factors of maximum instantaneous force are synchronizer life and the working strength requirement of shift execution mechanism. The main considering principle for determining maximum instantaneous force output by shift drive device contains:

\subsection{Meet the Requirement of Synchronizer Life}

Synchronizer life is the main consideration in the process of shift control strategy studying. The maximum instantaneous force loading on shift execution mechanism must meet the requirement of friction work per unit area of synchronizer friction lining. In addition, the rotary speed difference synchronous between input shaft and output shaft in the process of synchronization phase is depending on the friction function between the ring cone and engagement gear cone. The heat cased by friction function will make friction coefficient changed [6]. Under normal circumstances, the friction coefficient will become smaller in pace with the maximum instantaneous force becomes larger. The variation of friction coefficient should be one of refer factors of shift force loading rules formulating.

\subsection{Meet the Working Strength Requirement of Shift Execution Mechanism}

Transmission is the important component in automotive powertrain. For the convenience of the layout, each components of transmission should reduce the volume and the weight as much as possible under the premise of meeting design requirements. The key components are usually selected large strength safety factor in the design process of transmission prototype, such as each gear, input and output shaft, bearing, and so on. While some non-critical component, especially shift execution mechanism, the design parameters are usually determined by experience and bench test results. In such a process, the shift fork fracture is the common failure modes for shift execution mechanism [7, 8]. At this moment, the maximum instantaneous force output by shift drive device must to meet the working strength requirement of shift execution mechanism.

\section{MODELING AND MESHING FOR SHIFT FORK}

To determine the specific values of the maximum instantaneous force that the shift execution mechanism can bear during gear shift process, the three-dimensional model of the shift fork and the finite element analysis model based on ABAQUS are established. And then, the influence of strength and stiffness of shift fork on the shift force loading rules can be analyzed. The analysis results of maximum instantaneous force will be the consultation standard of the shift control strategy formulating. And the stiffness analysis can obtain variation curve of the maximum shift fork deformation in pace with the maximum instantaneous force, it will provided a good theoretical basis for reducing shift shock.

The shift mechanism of A-5-speed manual transmission is used as an example to illustrate the simulation, co-relation and validation of the shift fork strength and stiffness. The three-dimensional model of 4 gear shift fork is shown in
Fig. (1). In order to reduce the grid meshing difficulty and avoid an excessive amount of computation during analyzing, smaller size fillets and chamfers information that do not affect the finite element analysis results has been omitted in the model.

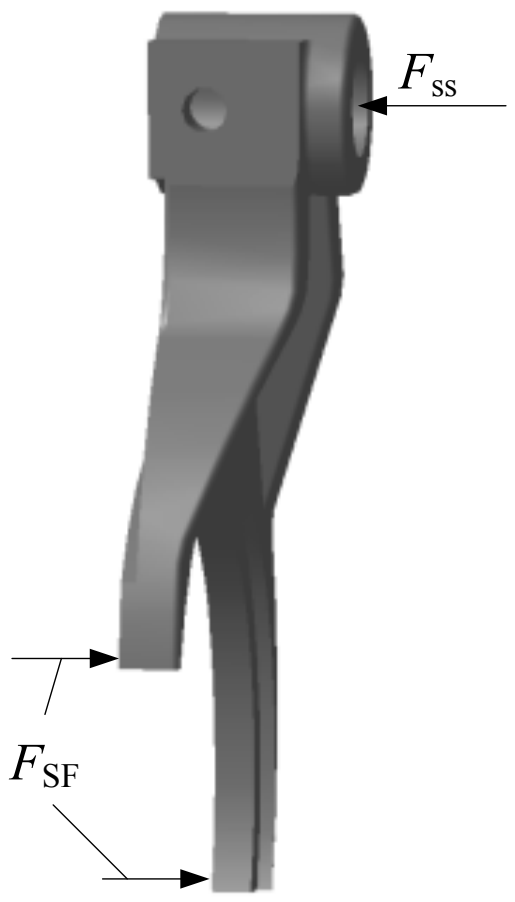

Fig. (1). Three-dimensional model of 4 gear shift fork.

In the Figure, $F_{\mathrm{ss}}$ is the shift force; $F_{\mathrm{SF}}$ is the axial reaction force existing shift fork legs.

The material used as shift fork mainly contains cast iron and aluminum in the industry. The aluminum material has a lower weight and a better wear characteristic, but it has a higher cost and a worse stiffness in the same time. Thus, the shift fork material of project studying is the common nodular cast iron in the industry. It has a better shock toughness, vibration damping, machinability, and the lower brittle transition temperature. The property parameter values of nodular cast iron are shown in Table 1 [9].

Table 1. Property parameter values of nodular cast iron.

\begin{tabular}{|c|c|}
\hline Parameter & Value \\
\hline \hline Tensile Strength /MPa & 400 \\
\hline Yield Strength /MPa & 250 \\
\hline Elastic Modulus /GPa & 157 \\
\hline Poisson ratio & 0.27 \\
\hline
\end{tabular}

Put the three-dimensional model of 4 gear shift fork into the workspace of ABAQUS and set the property parameter values of nodular cast iron into the material characteristic box of the software. And then, mesh the grid using free meshing technology in the software of ABAQUS, as shown in Fig. (2). 


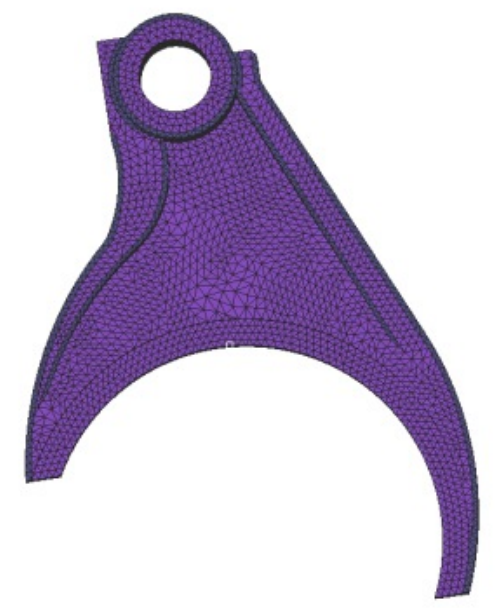

Fig. (2). Grid meshing result of shift fork.

To restrict all the degrees of freedom, set the displacement constraints at the shaft hole of shift fork. At the same time, add force load at the shift fork legs. The strength and stiffness analysis may be completed at this point.

\section{SIMULATION RESULTS}

The linear motion of shift fork can complete downshift and upshift operation, the maximum instantaneous force needed during downshift process is much less than upshift process. The shift fork finite element analysis of the strength and stiffness is only for upshift process.

When the axial reaction force named $F_{\mathrm{SF}}$ is $500 \mathrm{~N}, 1000$ $\mathrm{N}, 1500 \mathrm{~N}$ respectively, the strength analysis results are shown in Fig. (3) and the stiffness analysis results are shown in Fig. (4).

In the Figure, $\sigma_{\max }$ is maximum contact stress; $\sigma_{\mathrm{M}}$ is Von Mises stress.

According to the fourth strength theory, component distortion energy is the main factor to cause material yield. No matter how the stress state is, the material will generate yield when the shape energy reaches certain limit value that related to the material property $[10,11]$. At this time, the force status of shift fork must meet the requirement of Von Mises yield condition, scilicet, the maximum Von Mises stress of each unit existing in the component must be less than the yield strength of the nodular cast iron. Assuredly, the maximum contact stress must be less than the value of tensile strength too.

In the Figure, $\Delta S_{\max }$ is the maximum shift fork deformation. As can be seen from Fig. (4), the maximum shift fork deformation occurs in the shift fork legs.

The maximum contact stress, maximum Von Mises stress and maximum deformation of the shift fork are shown in Table 2 when the drive device output different shift force. We can see, the maximum contact stress is $135 \mathrm{MPa}$ and the maximum value of Von Mises stress is $210.88 \mathrm{MPa}$ when the shift force output by drive device is $1000 \mathrm{~N}$. The maximum contact stress and Von Mises stress are all meet the strength requirement of the shift fork material. At this moment, the maximum deformation of shift fork is $0.52 \mathrm{~mm}$. (a) $F_{\mathrm{SF}}=500 \mathrm{~N}$

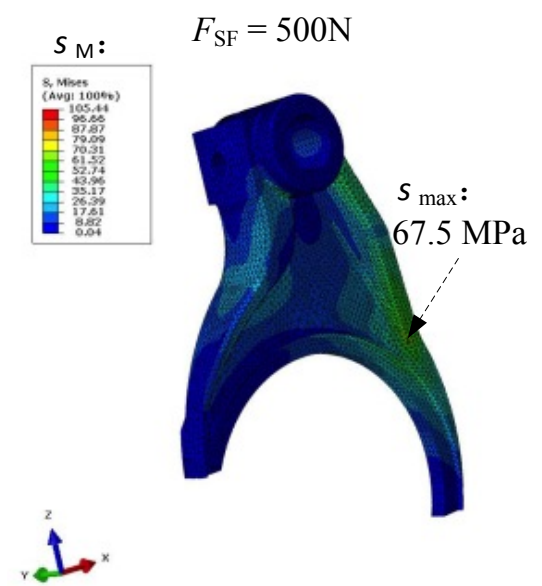

(b) $F_{\mathrm{SF}}=1000 \mathrm{~N}$
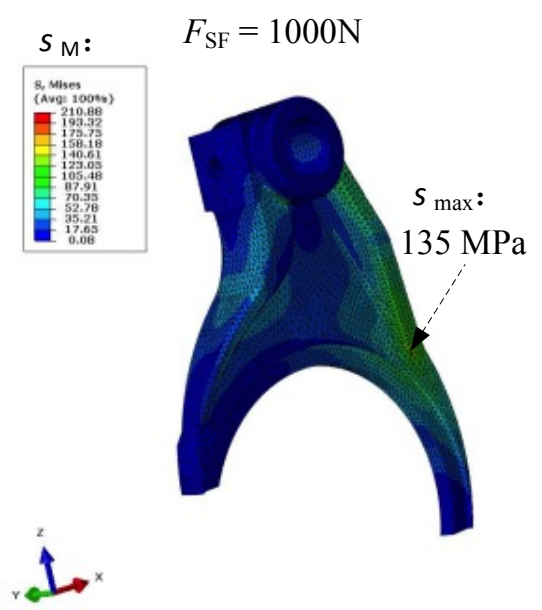

(c) $F_{\mathrm{SF}}=1500 \mathrm{~N}$

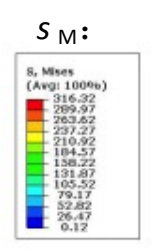

$F_{\mathrm{SF}}=1500 \mathrm{~N}$
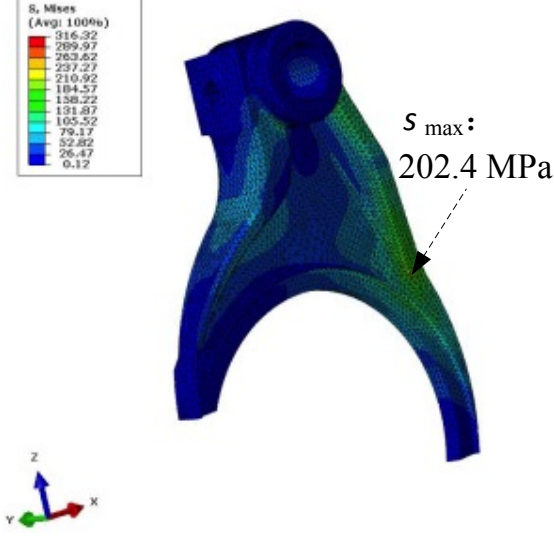

Fig. (3). The strength analysis results.

When the shift force output by drive device is $1500 \mathrm{~N}$, the maximum contact stress is $203.8 \mathrm{MPa}$ and the maximum value of Von Mises stress is $316.24 \mathrm{MPa}$. Von Mises stress can not meet the yield strength requirement of the shift fork material. It will easy to make the shift fork generate fatigue fracture at the place of maximum contact stress. 
(a) $F_{\mathrm{SF}}=500 \mathrm{~N}$

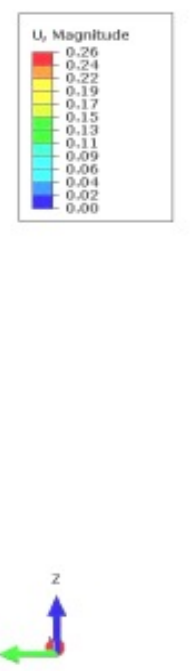

(b) $F_{\mathrm{SF}}=1000 \mathrm{~N}$

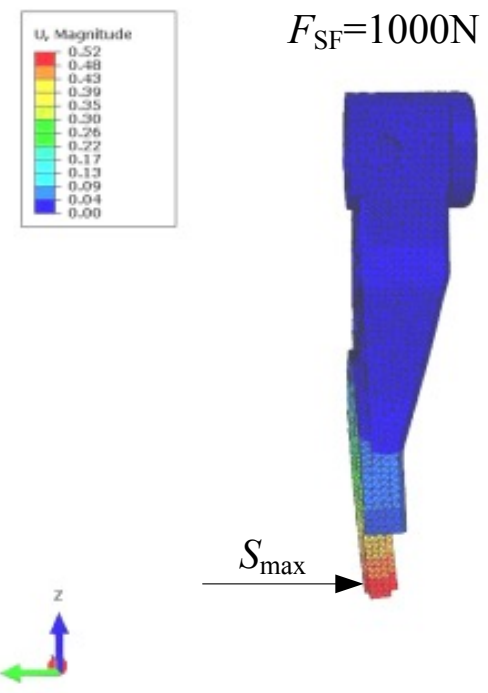

(c) $F_{\mathrm{SF}}=1500 \mathrm{~N}$

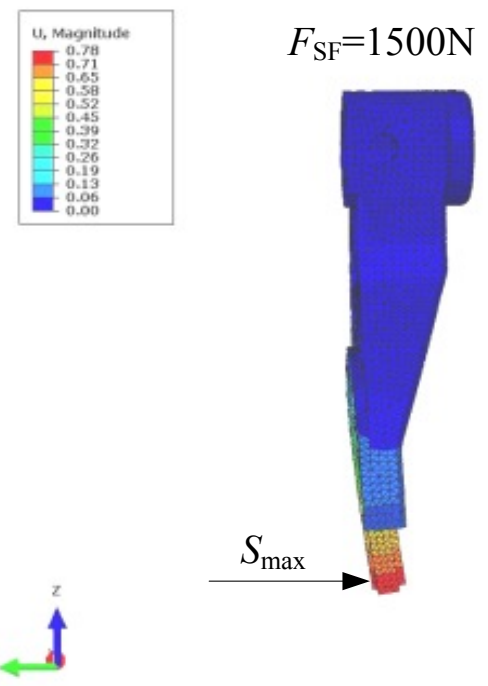

Fig. (4). The stiffness analysis results.
Table 2. Stress and deformation under different shift force affecting.

\begin{tabular}{|c|c|c|c|}
\hline $\boldsymbol{F}_{\text {ss }} / \mathbf{N}$ & $\sigma_{\max } / \mathbf{M P a}$ & $\sigma_{\text {Mmax }} / \mathbf{M P a}$ & $\Delta \boldsymbol{S}_{\max } / \mathbf{m m}$ \\
\hline \hline 500 & 67.5 & 105.44 & 0.26 \\
\hline-500 & 67.5 & 105.41 & 0.26 \\
\hline 1000 & 135 & 210.88 & 0.52 \\
\hline-1000 & 135 & 210.83 & 0.52 \\
\hline 1500 & 202.4 & 316.32 & 0.78 \\
\hline-1500 & 203.8 & 316.24 & 0.78 \\
\hline
\end{tabular}

In the Table, $\sigma_{\mathrm{M} \max }$ is the maximum value of Von Mises stress.

After repeated finite element simulation analysis, the variation curves for the maximum Von Mises stress value and the maximum deformation are summarized in pace with the shift force increasing, as shown in Fig. (5).

In the Figure, $\left[\sigma_{\mathrm{M}}\right]$ is the allowable value of Von Mises stress; $\left[F_{\mathrm{ss}}\right]$ is the allowable value of shift force provided by drive device; $\Delta S_{\max -\mathrm{M}}$ is the shift fork deformation value when shift force reaches allowable value.
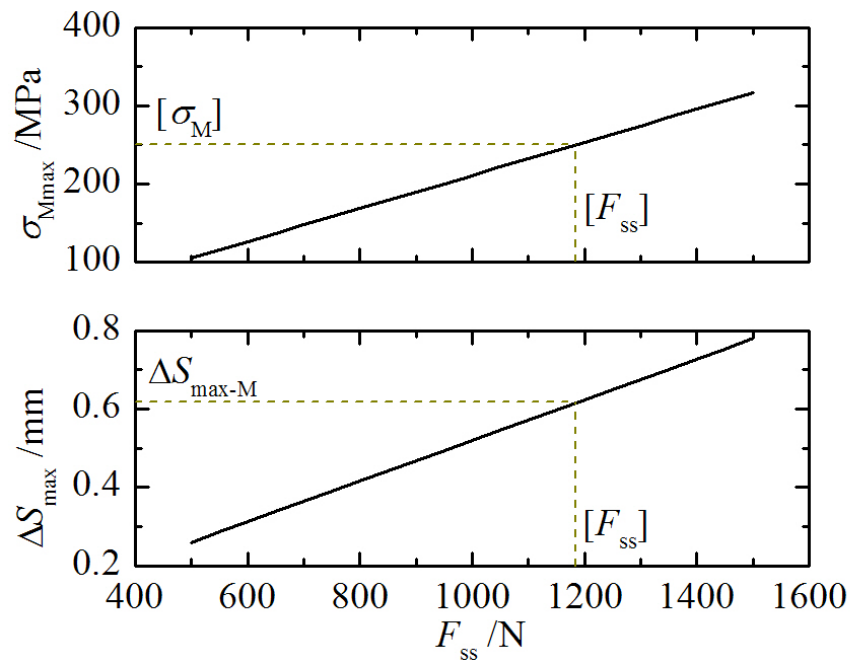

Fig. (5). Variation curves for Von Mises stress and maximum deformation.

We can see from Fig. (5), to meet the Von Mises stress requirement of nodular cast iron, the maximum instantaneous force output by shift drive device can not larger than $1185.5 \mathrm{~N}$ during the process of gear shift. In this time, the maximum shift fork deformation is $0.617 \mathrm{~mm}$ and the stiffness shift fork is $1.921 \times 10^{3} \mathrm{~N} / \mathrm{mm}$. It provides a sufficient theoretical basis for shift control strategy formulating.

\section{CONCLUSION}

Aiming to the lower system reliability and the higher shift shock of AMT, the shift force loading rules has been researched on the basis of strength and stiffness analyzing of shift fork. Formulation principle of maximum instantaneous force is proposed and the finite element analysis model based on ABAQUS is established. And then, the simulation 
analysis has been completed. It provides a sufficient theoretical basis for shift control strategy formulating and presents a good application prospect for AMT.

\section{CONFLICT OF INTEREST}

The authors confirm that this article content has no conflict of interest.

\section{ACKNOWLEDGEMENTS}

This work is a project supported by Shandong Provincial Natural Science Foundation (ZR2014EEQ031) and Shandong Province Science and Technology Development Program (2014GGX103007), China.

\section{REFERENCES}

H. Naunheimer, B. Bertsche, J. Ryborz, and W. Novak "Automotive Transmissions Second Edition," Heidelberg: Springer Verlag, 2010.

[2] H. Liu, Y. Lei Z. Li, J. Zhang, and Y. Li "Gear-shift strategy for a clutchless automated manual transmission in battery electric vehicles," SAE 2012 World Congress, Warrendale PA, SAE, 201201-0115, 2012.
[3] W. Ge, B. Li, Y. Zhao, Z. Liu "AMT shift system based on directdrive and self-energizing technology," Transactions of the Chinese Society for Agricultural Machinery, vol. 45, no. 9, pp. 1-7, 2014.

[4] Z. Zhong, G. Kong, Z. Yu, X. Chen, X. Chen, and X. Xin, "Concept evaluation of a novel gear selector for automated manual transmission," Mech Syst Signal Process, vol. 31, pp. 316 331, 2012.

[5] R. Kunal, "Gear Shift Fork Stiffness Optimisation". SAE 2011 World Congress, Warrendale PA, SAE, 2011-01-2235. 2011.

[6] M. Spreckels, "Influence of temperature distribution on the tribological performance of automotive synchronizers," Hannover: University of Hannover, 2001.

[7] D.M. Chen, X.Y. Li, J.B. Hu, and S.H. Yuan "Fracture of ShiftingYoke in the Gear-Box for a Wheeled Chariot Based on Miner's Accumulated Damage Theory," Transactions of Beijing Institute of Technology, vol. 26, no. 10, pp. 855-858, 887, 2006.

[8] Y.G. Zhou, N. Guo, G. Lei, J.M. Liu, and Z.Z. Zhao, "Fracture Analysis of Shifting-Yoke in Gear-Box with Direct Push Cylinder," Journal of Chongqing Institute of Technology (Natural Science), vol. 22, no. 7, pp. 14-16, 25, 2008.

[9] D. X. Cheng, "Mechanical Design Handbook," Beijing: Chemical Industry Press, 2004

[10] Z. Q. Hu, L. Guo, X. Y. Jiang, et al. "Mechanics of Materials", Beijing: Higher Education Press, 2009.

[11] M. X. Chen, "Elastic-Plastic Mechanics," Beijing: Science Press, 2007.

(C) Bo et al.; Licensee Bentham Open.

This is an open access article licensed under the terms of the (https://creativecommons.org/licenses/by/4.0/legalcode), which permits unrestricted, non-commercial use, distribution and reproduction in any medium, provided the work is properly cited. 